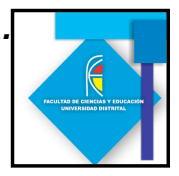

\title{
IMPLEMENTACIÓN DE LAS TIC EN LA ENSEÑANZA DE LA FÍSICA, FACTIBILIDAD Y EFICACIA EN NUESTRO PAÍS.
}

\section{IMPLEMENTATION OF TIC IN TEACHING PHYSICS, FEASIBLE AND EFFECTIVE IN OUR COUNTRY}

\author{
Jhon Raul Osorio Meneses ${ }^{1}$ \\ Miguel Antonio Prieto Murcia² \\ Esperanza Del Pilar Infante Luna ${ }^{3}$
}

\section{Resumen}

Desde hace algunas décadas se han venido incorporando las Tecnologías de la Información y la Comunicación (TIC) como complemento del material educativo, con lo que se esperaba un fuerte impacto en los métodos de enseñanza, revolucionando así la educación tanto escolar como universitaria. (Eduteka, 2007)

A partir del surgimiento de la web y su uso en la enseñanza se especulaba una transformación educativa en países desarrollados e incluso en los países del tercer mundo (Eduteka, 2007); no obstante algunos autores hablan de un aumento en la brecha entre el nivel educativo de las escuelas latinoamericanas y las escuelas que hacen parte de los miembros de la Organizaciones para la Cooperación y el Desarrollo Económico. Con el fin de hacer una evaluación de la implementación de las TIC en la enseñanza y en particular en la enseñanza de la física en Colombia, se recopiló información reportada por diferentes autores en relación con el tema, la cual fue sistematizada y analizada. En este artículo se presentan los resultados de dichos análisis y a partir de estos se plantean algunos aspectos asociados con la eficacia y la factibilidad del empleo de las Tic en nuestro país, los cuales pueden ser tenidos en cuenta tanto por investigadores como por usuarios de estas tecnologías.

Palabras clave: TIC, enseñanza, escuela, material educativo.

\footnotetext{
Abstract

For some decades they have come incorporating the Technologies from the Information and the Communication (TICs) to complement the educative material, and so a fort was expected as much impact in the methods of education revolutionizing therefore the

${ }^{1}$ Estudiante Proyecto curricular de licenciatura en Física - U.D. Profesora Proyecto curricular de licenciatura en Física U.D. Grupo de investigación Física Aplicada a las Ciencias Bilógicas (FIACIBI). jrosoriom@correo.udistrital.edu.co

${ }^{2}$ Estudiante Proyecto curricular de licenciatura en Física - U.D. Profesora Proyecto curricular de licenciatura en Física U.D. Grupo de investigación Física Aplicada a las Ciencias Bilógicas (FIACIBI) kailazcarot@yahoo.com

${ }^{3}$ Estudiante Proyecto curricular de licenciatura en Física - U.D. Profesora Proyecto curricular de licenciatura en Física U.D. Grupo de investigación Física Aplicada a las Ciencias Bilógicas (FIACIBI) epinfantel@udistrital.edu.co
} 


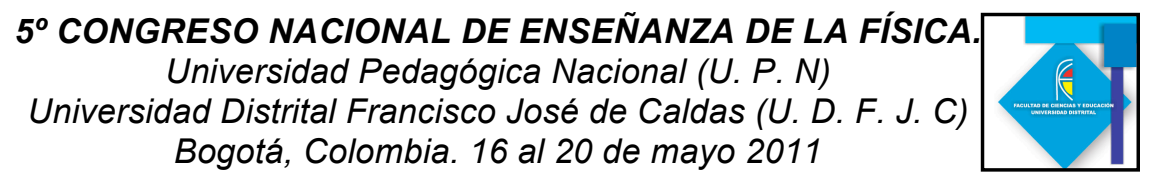

education student as university. (Eduteka, 2007) From the sprouting of the Web and its use in education an educative transformation in developed countries and in the countries of the Third World was even speculated on (Eduteka, 2007); despite some authors the educative level of the Latin American school and the school in Member States of the Organization for the Economic Cooperation and the Development speak of an increase in the breach between. With the purpose of in particular to make an evaluation of the implementation of the TICs in education and in education of the physics in Colombia, information reported by different authors in relation to the subject was compiled, which was systematized and analyzed. In this article the results of this analysis appear and from these some aspects associated with the effectiveness and the feasibility of the use of the Tics in our country consider, which can as much be considered by investigators as by users of these technologies.

Keywords: TIC, education, school, educative material.

\section{Introducción. Tecnología y educación}

Cuando se habla de las nuevas tecnologías de la información y comunicación en la sociedad más conocidas como TIC, es inherente hablar de la íntima relación que estas tienen con la educación, lo anterior obedece al hecho de que cada elemento que ha facilitado las formas de comunicación entre las personas a su vez ha suscitado grandes cuestionamientos en lo referente a su implementación en los procesos de transmisión de conocimiento.

Tal es el caso del uso del periódico, la radio, el cine, la televisión, y el video; sin embargo estos medios no lograron colmar las expectativas que se generaron en torno a su implementación en educación, siendo casi irrelevantes y usados solamente en casos esporádicos (Moreno, 2003).

Contrario a ello en los últimos años con las llamadas Redes de comunicación y la World Wide Web $(w w w)$ y su elemento más extenso la Internet, han ocurrido verdaderos cambios en las formas de enseñanza debido a que permiten al usuario interactuar y comunicarse de forma más rápida y económica que cualquier otro medio de comunicación, lo que las ha convertido en una herramienta a emplear de uso frecuente en educación.

Adicionalmente los cambios de las costumbres familiares y los nuevos valores introducidos en la sociedad generaron un impacto particular sobre los estudiantes que crecieron en un ambiente de transformaciones tecnológicas importantes que afectaron, cada una a su tiempo, las estructuras de comunicación dentro de la familia y de la sociedad.

Debido a esto las nuevas generaciones de jóvenes estudiantes, contrario a las pasadas generaciones, están llenas de expectativas y necesidades diferentes que se manifiestan en los diferentes ambientes de enseñanza (Thierry Karsenti, 2001). 


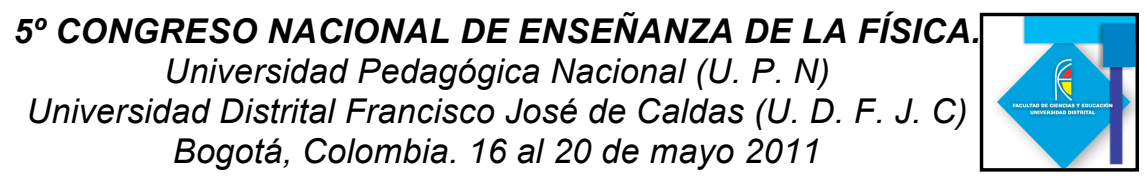

Teniendo en cuenta lo anterior surgen nuevos desafíos a los que se enfrenta la pedagogía en las diferentes modalidades y niveles de enseñanza, particularmente en lo que se refiere

a la motivación y al desarrollo de medios tecnológicos integrados a los ambientes de educación y de aprendizaje que realmente contribuyan a los procesos que se viven en el aula y fuera de esta.

Sin embargo es necesario evaluar las ventajas y desventajas que ofrece la implementación de las TIC en la enseñanza de la física, así como su factibilidad y eficacia en nuestro país como vía académica no tradicional. En este artículo se presenta la metodología y resultados obtenidos del análisis realizado a partir de la información recopilada a través de diferentes medios como artículos científicos y tesis tanto de pregrado como de posgrado; mostrando las diferentes miradas que tienen los autores sobre las tecnologías de la información y comunicación y su implementación en los procesos de enseñanza aprendizaje tanto dentro como fuera del aula de clase.

\section{Metodología}

Como primera medida teniendo en cuenta la naturaleza de la investigación se definieron las fuentes de información a ser consultadas, estableciendose las bases de datos Dialnet y PROQUEST, la cual a su vez se divide en ERIC\& y ProQuest Education Journals, de fácil acceso en la U. Distrital, también consideramos fundamentales las tesis tanto de pregrado como de posgrado de las universidades Pedagógica y Distrital de Bogotá, esta información se complementó con una búsqueda en google académico.

Al acceder a estas bases de datos se utilizaron como criterios de búsqueda las siguientes palabras clave: TIC, educación, ambiente virtual, enseñanza, escuela, material educativo, cursos virtuales. Con el fin de organizar la información para su posterior análisis esta fue sistematizada de acuerdo con las siguientes categorías: Autor(es), título del documento, ubicación (base de datos, bibliotecas internet), fecha de publicación y fecha de consulta en caso de tratarse de un documento de la red, y resumen del documento.

Finalmente teniendo en cuenta que uno de los objetivos de nuestra investigación es establecer los factores que determinan la viabilidad y efectividad de la implementación de las Tic en nuestro país, se realizó un análisis de cada uno de los documentos recopilados haciendo énfasis en los siguientes aspectos: beneficios de las Tic (autonomía, espacio geográfico, cursos a distancia) y requerimientos mínimos para su implementación; este análisis fue consolidado junto a las categorías antes establecidas. Finalmente se realizó un análisis en conjunto de la información sistematizada.

\section{Análisis de la información}

A continuación se presentan los aspectos que consideramos relevantes luego del análisis realizado con base en los parámetros ya mencionados. Uno de los factores de mayor interés en el uso de las Tics está referido a los cursos a distancia ya que posibilitan la educación en lugares donde no es factible contar con docentes. Algunos autores plantean que la distancia entre el instructor y los estudiantes no aumenta ni disminuye la 


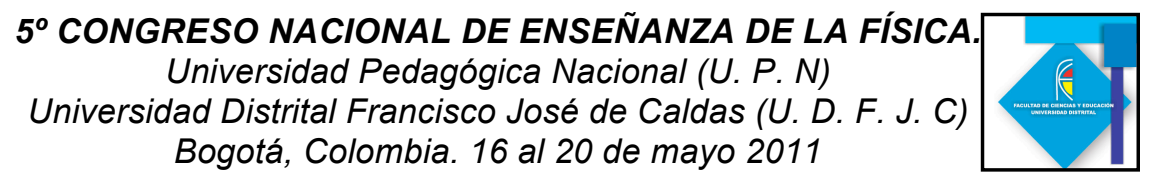

efectividad e interacción, ya que el enfoque está dado en la pedagogía de atraer a los estudiantes y no en la tic utilizada para entregar el contenido (Eduteka, Stelacio, \& Kietel , 2009).

Varios autores coinciden en que una de las mayores ventajas que tiene las tic es la manipulación de las herramientas en la solución de problemas y su progresivo desarrollo, lo cual no debe ser visto desde la perspectiva de un docente que enseña comandos, si no en relación a la facilidad que se tiene al momento de solucionar problemas y/o realizando cálculos numéricos complejos empleando estas tecnologías; sin embargo esto implica una actitud activa por parte del docente al momento de realizar actividades que permitan que estas destrezas se desarrollen dentro del ámbito virtual. (Figueroa, 2006)Otro factor que consideramos importante de tener en cuenta en relación con la implementación de las Tic, es el trabajo independiente de los estudiantes ya que el estudiante puede explorar de manera autónoma la información, definir enfoques en las áreas de su interés, y manejar su tiempo de manera flexible.

Es importante mencionar que una de las características de la enseñanza tradicional es la interacción entre los estudiantes y el docente, la investigadora Marguerita Mcvay identifica cuatro tipos de interacción: con el contenido, con el instructor, con los compañeros y consigo mismo, estos aspectos deben tenerse en cuenta al momento de implementar clases en un ambiente virtual ya que en estas, a diferencia de las clases tradicionales, cambian aspectos como el lenguaje y el espacio en el que se desarrolla la clase, por lo que se debe generar un ambiente ameno para permita la interacción de los participantes.

Finalmente dentro de los resultados reportados por el proyecto TACTICS (Técnicas de Aprendizaje Colaborativo con Tecnologías de Información y Comunicación en Ciencias) (Casanova, 2002) se identifican algunas desventajas de las tics, asociadas principalmente a la falta de recursos, lo que implica que las instituciones educativas deben mejorar su infraestructura tecnológica de forma tal que sea factible y eficiente la implementación de propuestas educativas en torno a las Tics, de forma tal que se obtenga una evaluación satisfactoria de las herramientas empleadas en clase. (Navarro, 2009)

\section{Discusión - Implementación de las tics en Colombia}

Hemos planteado diferentes perspectivas en torno a la implementación de las Tic en la educación, sin embargo es necesario ir un poco más allá en nuestro análisis y establecer los aspectos que consideramos relevantes al momento de poner en marcha proyectos de esta naturaleza en nuestro país.

El uso de nuevas tecnologías de la información y comunicación es una necesidad que día a día se ha ido incrementando, sin embargo su uso en educación está limitado al cumplimiento de unos requisitos tanto de software como de hardware (Casanova, 2002), que deben ser tenidos en cuenta en las instituciones educativas de nuestro país al momento de considerar su implementación.

De acuerdo con los aspectos analizados es posible afirmar que dadas las características geográficas y socioeconómicas de nuestro país la implementación de cursos virtuales 


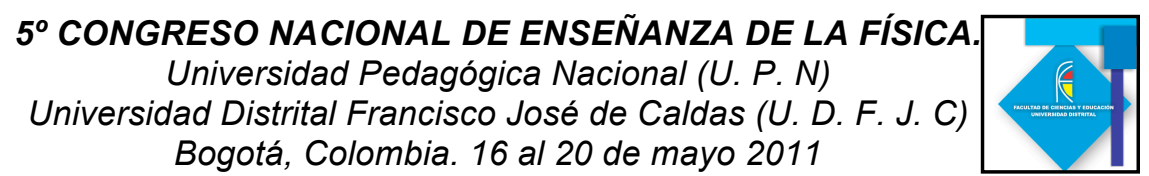

resulta ser una propuesta viable que suple carencias como falta de docentes e instituciones educativas en regiones apartadas de nuestro país. Sin embargo la implementación de nuevas tecnologías en el aula de clase, dados sus requerimientos tecnológicos, debe ser analizada con más cuidado o de lo contrario se podría convertir en recursos adquiridos que no pueden ser empleados debido a limitaciones de hardware $y / 0$ de recursos como conexiones a internet.

Finalmente es de mencionar que diferentes autores coinciden en el papel que juega el docente al momento de implementar en el aula de clase nuevas tecnologías, ya que el lenguaje, el espacio y las dinámicas en que se desarrollan las clases "virtuales" es muy diferente al tradicional.

\section{Referencias Bibliográficas}

Casanova, G. W. (2002). Redie. Recuperado el 11 de 04 de 2010, de Revista Electrónica de Investigación Educativa: http://redie.ens.uabc.mx/vol4no1/contenido-waldegg.html

Cuban, L. (1986). Teachers and machines: The classroom use of technology since 1920. Teachers College Press., 19.

Eduteka. (01 de septiembre de 2007). http://www.eduteka.org/. Recuperado el 1 de Marzo de 2010, de http://www.eduteka.org/PorQueTIC.php:

http://www.eduteka.org/PorQueTIC.php

Eduteka, Stelacio, D., \& Kietel , j. (01 de 12 de 2009). Eduteka. Recuperado el 12 de 04 de 2010, de http://www.eduteka.org/imprimible.php?num=1027

Figueroa, W. (16 de 12 de 2006). un caso exitoso de enseñanza de las tic. (Eduteka, Entrevistador)

Ministerio Nacional De Educación. (2006). http://www.plandecenal.edu.co/. Recuperado el 21 de Marzo de 2010, de http://www.plandecenal.edu.co/html/1726/w3-channel.html: http://www.plandecenal.edu.co/html/1726/w3-channel.html

Moreno, J. G. (2003). Plataformas digitales y sus. Revista Complutense de Educación Vol. 14 Núm. 2, 563-588.

Navarro, J. (2009). Elaboración de una herramienta para la evaluación de Software Educativo de Mecánica Clásica. Bogota.

Thierry Karsenti, F. L. (2001). Les TIC au coeur de la pédagogie universitaire :. En F. L. Thierry Karsenti, Les TIC au coeur de la pédagogie universitaire : (págs. 174 - 177). Québec: Presses de I/'Université du Québec. 